\title{
Parents' Knowledge and Practice of Iron Therapy for Anemic Infants in Primary Health Care in Bahrain
}

\author{
Ahmed Al-Jamri ${ }^{1}$, Fatema Al-Awainati ${ }^{1}$, Marwa Ali $^{1}$, Masooma Jaafar ${ }^{1}$, Mustafa Hussain ${ }^{1} \&$ Ghufran Jassim ${ }^{2}$ \\ ${ }^{1}$ Family Physician, Ministry of Health, Juffair, Kingdom of Bahrain \\ ${ }^{2}$ Senior Lecturer in Family Medicine, RCSI Bahrain, Busaiteen, Kingdom of Bahrain \\ Correspondence: Ghufran Jassim, Senior Lecturer in Family Medicine Department, RCSI Bahrain. P.O. Box: \\ 15503, Adliya, Bahrain.
}

Received: January 27, 2017 Accepted: February 20, 2017 Online Published: April 6, 2017

doi:10.5539/gjhs.v9n8p99 URL: https://doi.org/10.5539/gjhs.v9n8p99

\begin{abstract}
Background: Anemia in children aged less than five years is a common medical problem worldwide. Iron deficiency anemia is the most prevalent anemia in infants and has been shown to be a public health problem. Many studies have been conducted on anemia among infants but the scope of the conducted studies was focused on the prevalence of anemia with less emphasis on the knowledge and parental practice of anemia and its treatment. Providing infants with iron supplements and simultaneously ensuring their compliance has shown an improvement in hemoglobin levels, along with a significant reduction in the prevalence of iron deficiency anemia (IDA).
\end{abstract}

Objective: To explore the level of parental knowledge and practice towards iron syrup.

Methods: A Cross- sectional study was carried out from $21^{\text {st }}$ June to $2^{\text {nd }}$ July, 2015 in ten Primary Health Care centers in Bahrain. During the study period, a phone survey was conducted using a self-constructed questionnaire. The response rate was $98 \%$.

Results: In this study, the prevalence of anemia in infants is $45 \% .58 .4 \%$ of parents did not give the offered iron for their infants or stopped it within the first week of use. Overall, around $70 \%$ of parents correctly answered half or more of the knowledge questions regarding iron syrup side effects but only $32.5 \%$ of parents correctly answered half or more of the questions regarding IDA complications.

Conclusion: Anemia is prevalent among 9 months old infants with considerable gaps in knowledge and practices of parents.

Keywords: Anemia, Knowledge, Practice, Primary Health Care, Kingdom of Bahrain

\section{Introduction}

Anemia is a condition in which the number of red blood cells or their oxygen carrying capacity is insufficient to meet the physiological needs, which vary by age, gender, attitude, smoking and pregnancy (WHO, 2015). Anemia in children (below 5 years old) is a common medical problem worldwide (WHO, 2015) and it is defined as a hemoglobin level $<11 \mathrm{~g} / \mathrm{dl}$ according to the World Health Organization (UNICEF/UNU/WHO, 2001). Iron deficiency anemia (IDA) is the most prevalent anemia in infants and has been shown to be a public health problem that affects low-, middle- and high-income countries (WHO, 2015).

Globally, the prevalence of anemia in children less than 5 years of age was $42.6 \%$ in 2011 (WHO, 2015). In the same year, in Gulf Corporation Countries (GCC), it ranged from 26\% in Qatar and Kuwait to 41\% in Oman (WHO, 2015). In Bahrain, according to annual statistics from the Ministry of Health in 2012, the prevalence of anemia was $31 \%$ among infants in primary health centers (Ministry of Health). In addition, the few studies which have been conducted locally on anemia among infants were aimed at determining the prevalence of anemia, which has ranged between 40.5\% to 49.6\% (Ahmed G, September 2006; Al-Alawi F, March 2015; Al-Alawi M, 2014).

Worldwide, several studies have been conducted to investigate the knowledge of Iron Deficiency Anemia (IDA) or practice with the treatment (Abdinia, April 2014; Bilenko, Yehiel, Inbar, \& Gazala, 2007; F Salehi, 2015; Hassan, Kamal, Fetohy, \& Turky, 2005; Talebi, Parisay, \& Mokhtari, 2012). Some studies in the Middle East, parents showed low to moderate knowledge of the disease and its treatment (Bilenko et al., 2007; Talebi et al., 2012). Regionally, a study was conducted in the United Arab Emirates which showed that only $18.0 \%$ of mothers had a 
satisfactory level of knowledge (Hassan et al., 2005).

Regarding parental practice, compliance was reported to be low in one study conducted in Israel (Bilenko et al., 2007). In Iran, two studies showed poor levels of parental practice and attitudes towards iron therapy for their infants (F Salehi, 2015; Talebi et al., 2012). In Bahrain, a recent study was conducted in one health centre and revealed only a 7.6\% compliance rate (Al-Alawi F, March 2015). However, the comparability of these studies is by far very complex due to the variability in the instruments used and the populations included.

Literature consistently documents the long term developmental outcomes of IDA during infancy. Recent studies have yielded significant association between IDA and poor cognition, school achievement and behavioral problems (Carter et al., 2010; Lozoff et al., 2008; Sherriff, 2001; Williams et al., 1999). However, iron therapy can be accompanied by bothersome side effects that might affect compliance (Talebi et al., 2012). Understanding parents' knowledge and perception towards the treatment might help address the issues that compromise their compliance.

Providing infants with iron supplements and simultaneously ensuring their compliance has shown an improvement in hemoglobin levels, along with a significant reduction in the prevalence of IDA (Coutinho, Goloni-Bertollo, \& Pavarino-Bertelli, 2008). Two regional studies have revealed similar significant improvements in hemoglobin levels in children of mothers who were assigned to an interventional health education program about IDA (Al-Alawi F, March 2015; Hassan et al., 2005).

The initial objective of this study is to explore the level of parental knowledge and practice towards iron syrup. Our hypothesis is that parents of anemic infants have scant knowledge and poor practice regarding iron treatment.

Addressing the gap in knowledge and practice towards IDA treatment might help setting strategies and interventions which may improve parental compliance and eventually lead to our ultimate goal, which is reducing the prevalence of IDA and its complications in Bahrain.

\section{Methods}

\subsection{Study Settings}

In the Kingdom of Bahrain, primary health care services are provided through 27 health centers distributed among 5 health regions all over the Kingdom (Al-Alawi M, 2014). A maternal and child health department (MCH) in each health center provides periodic child screening, including capillary hemoglobin $(\mathrm{Hb})$ measurement at nine months, 18 months, three years and five years of age, as recommended by the National Child Screening Guidelines (Al-Alawi F, March 2015).

\subsection{Study Design}

This is a cross-sectional study.

\subsection{Data Collection}

Data was collected over two weeks, from $21^{\text {st }}$ June to $2^{\text {nd }}$ July, 2015 in the primary health care centers in the Kingdom of Bahrain. Data (using a structured questionnaire tool) was obtained from the parents of eligible infants through phone calls.

\subsection{Data Collection Tool}

The data collection tool was based on medical references and previous literatures (Al-Alawi F, March 2015; Carter et al., 2010; Hassan et al., 2005; Lozoff et al., 2008; Sherriff A, 2001; Talebi et al., 2012; Williams et al., 1999). The content validity was tested via two pediatric consultants working in Ministry of Health. The questionnaire is composed of three parts: the first part is about the sociodemographic data of the parents. The second part includes questions to explore the knowledge of parents about iron deficiency anemia treatment and complications through listing true and false statements. The last part assessed parents' practices including their compliance to the prescribed iron syrup and their opinions regarding the importance of iron syrup and its side effects.

\subsection{Pilot Study}

A pilot study was conducted to test the language simplicity and comprehension of the questionnaire on 20 participants. Based on the pilot study, some modifications were made to the questionnaire. Furthermore, a training session for the interviewers was arranged to standardize the questions and to limit the impact of variability among the interviewers.

\subsection{Sample Size}

The calculated sample size for the study was up to 385 , which was determined using an estimated proportion of 0.5 
which yielded the maximum sample size, because information about the upper bound of the proportion was not known.

\subsection{Sampling Technique}

Health care centers were stratified into 5 strata that represented the health regions they belong to. Two Health Centers were randomly selected from each stratum. A proportionate sample was taken from each Health Center based on the number of anemic infants born in June, July and August 2014 in that Health Center (Figure 1).

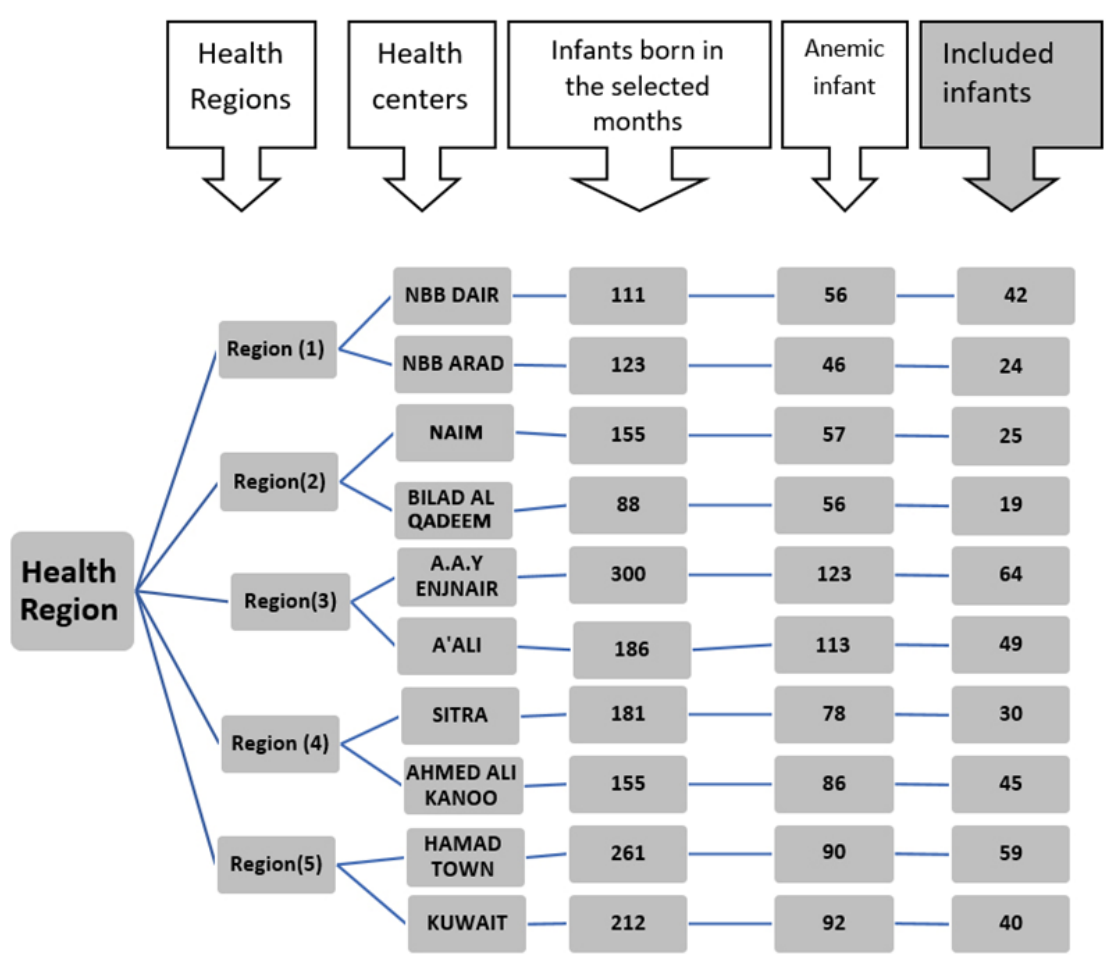

Figure 1. Sample selection

\subsection{Study Subjects}

All screening booklets of infants born in June, July and August 2014 (1772 booklets) were reviewed in the selected primary health care centers during the period of data collection. Parents of infants registered in the selected primary health centers were eligible if their infants had a $\mathrm{Hb}$ level $<11 \mathrm{~g} / \mathrm{dl}$ and had been offered iron syrup for IDA at the age of nine months. Parents had to speak either Arabic or English.

\subsection{Exclusion Criteria}

Caregivers other than parents or those who could not be reached after at least three phone calls at different times (Morning and evening) during the 2-week period of data collection were excluded.

All screening booklets from each Health Centre were reviewed for two things, low $\mathrm{Hb}(<11 \mathrm{~g} / \mathrm{dl})$ and a management plan. Because of poor documentation relating to a management plan, we needed to call all parents of anemic infants to check whether they were offered iron syrup by their primary care physician (Figure 2).

\subsection{Data Management and Analysis Plan}

The collected data was analyzed using SPSS version 22. Frequency and percent distributions were computed for the categorical variables while means and standard deviations were computed for the quantitative variables.

\subsection{Ethical Consideration}

Ethical issues have been considered carefully, verbal informed consent was taken at the beginning of each interview. This study was approved by the Research Committee of Ministry of Health in Bahrain. 


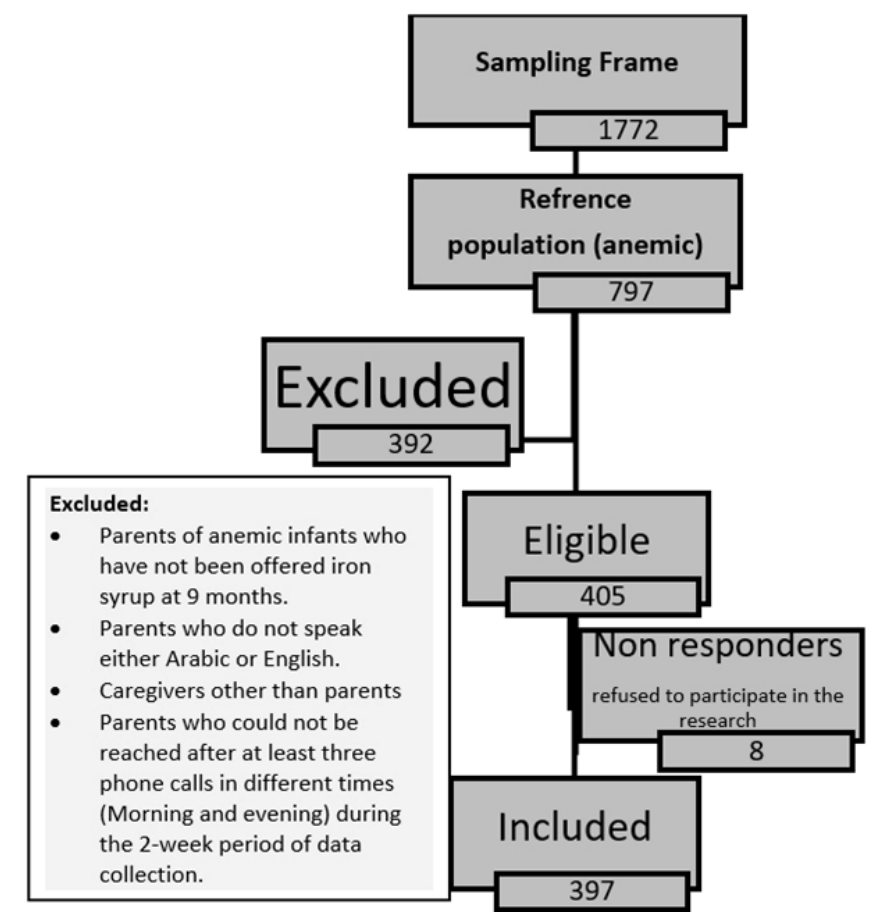

Figure 2. Study flow chart

\section{Results}

\subsection{Demographic Data}

The total number of infants in the selected three months was 1772 and those with low Hemoglobin level were 797 which reflect a prevalence of $45.0 \%$ (Figure 2). Three hundred and ninety-seven parents were included in the study, the majority were mothers $(95 \%)$, Bahraini $(89.2 \%)$, highly educated (University or equivalent) $(61 \%)$ and unemployed $(61 \%)$ (Table 1). The mean $\pm \mathrm{SD}$ of parental age and number of children per parents were 30.6 (SD 5.9 ) and 2.6 (SD 1.5) respectively.

\subsection{Knowledge}

$82.9 \%$ of all parents enrolled in the study said that iron syrup was given as treatment of anemia, $14.6 \%$ said that it was a routine supplement. Regarding the knowledge of iron syrup's side effects, $75.6 \%$ of participants reported that it may cause teeth decay and $75.8 \%$ said that it causes teeth staining. The data showed that $80.9 \%$ reported that dark stools is a side effect of iron syrup and $56.9 \%$ were aware of constipation as a side effect (Table 2). Some parents said that untreated IDA may lead to psychological and behavioral problems $17.4 \%$, heart problems $18.1 \%$, and rickets $71.8 \%$ (Table 2).

Overall, around $70.0 \%$ of parents correctly answered half or more of the knowledge questions regarding iron syrup side effects. $32.5 \%$ of parents correctly answered half or more of the questions regarding IDA complications.

$32.0 \%$ of parents obtain the information about IDA treatment and complications mainly from healthcare professionals (doctors or nurses), $28.5 \%$ from mass media, $24.4 \%$ from relatives and friends, $7.6 \%$ from health educational leaflets, and $3.8 \%$ from past experience.

$70.0 \%$ of parents said that it is possible to overcome the side effects (Table 3 ). $73.0 \%$ of parents reported that iron syrup is necessary to treat IDA. The majority of parents $(85.9 \%)$ said that a diet rich in iron is enough to replace iron therapy (Table 3). 
Table 1. Frequency and percent distribution of participants' demographical data $(n=397)$

\begin{tabular}{|c|c|c|c|}
\hline Demographic Data & Categories & n & $\%$ \\
\hline \multirow[t]{2}{*}{ The Participant } & Father & 20 & 5.0 \\
\hline & Mother & 377 & 95.0 \\
\hline \multirow[t]{3}{*}{ Age } & $<25$ & 65 & 16.5 \\
\hline & $25-35$ & 250 & 63.5 \\
\hline & $>35$ & 79 & 20.1 \\
\hline \multirow[t]{2}{*}{ Nationality } & Bahraini & 354 & 89.2 \\
\hline & Non-Bahraini & 43 & 10.8 \\
\hline \multirow[t]{3}{*}{ Educational Level } & Below secondary & 37 & 9.3 \\
\hline & Secondary & 118 & 29.7 \\
\hline & University or Equivalent & 242 & 61.0 \\
\hline \multirow[t]{2}{*}{ Occupation } & Employed & 155 & 39.0 \\
\hline & Unemployed & 242 & 61.0 \\
\hline \multirow[t]{3}{*}{ Income per month in BD } & $<500$ & 165 & 41.9 \\
\hline & $500-<1000$ & 159 & 40.3 \\
\hline & $1000+$ & 70 & 17.8 \\
\hline \multirow[t]{4}{*}{ Number of Children } & 1 Child & 108 & 27.2 \\
\hline & 2 Children & 106 & 26.7 \\
\hline & 3-4 Children & 142 & 35.8 \\
\hline & $>4$ Children & 41 & 10.3 \\
\hline \multirow{2}{*}{$\begin{array}{l}\text { Have you ever been prescribed iron syrup for any of } \\
\text { your other children? }\end{array}$} & Yes & 179 & 45.1 \\
\hline & No & 218 & 54.9 \\
\hline
\end{tabular}

Table 2. Frequency and percent distribution of knowledge of the Parents (Iron therapy side effects and anemia complications) $(\mathrm{n}=397)$

\begin{tabular}{|c|c|c|c|c|}
\hline & \multicolumn{2}{|c|}{ Incorrect answer } & \multicolumn{2}{|c|}{ Correct answer } \\
\hline & $\mathbf{n}$ & $\%$ & $\mathbf{n}$ & $\%$ \\
\hline Delay in teething & 219 & $55.2 \%$ & 178 & $44.8 \%$ \\
\hline Teeth Staining & 96 & $24.2 \%$ & 301 & $75.8 \%$ \\
\hline Teeth decay & 300 & $75.6 \%$ & 97 & $24.4 \%$ \\
\hline Walking difficulty & 174 & $43.8 \%$ & 223 & $56.2 \%$ \\
\hline Constipation & 171 & $43.1 \%$ & 226 & $56.9 \%$ \\
\hline Speech delay & 140 & $35.3 \%$ & 257 & $64.7 \%$ \\
\hline Dark stool & 76 & $19.1 \%$ & 321 & $80.9 \%$ \\
\hline Hair loss & 146 & $36.8 \%$ & 251 & $63.2 \%$ \\
\hline Poor concentration & 231 & $58.2 \%$ & 166 & $41.8 \%$ \\
\hline Jaundice & 234 & $58.9 \%$ & 163 & $41.1 \%$ \\
\hline Learning difficulty & 274 & $69.0 \%$ & 123 & $31.0 \%$ \\
\hline Growth delay & 242 & $61.0 \%$ & 155 & $39.0 \%$ \\
\hline Psychological and behavioral problem & 328 & $82.6 \%$ & 69 & $17.4 \%$ \\
\hline Cancer & 176 & $44.3 \%$ & 221 & $55.7 \%$ \\
\hline Heart problem & 325 & $81.9 \%$ & 72 & $18.1 \%$ \\
\hline Rickets & 285 & $71.8 \%$ & 112 & $28.2 \%$ \\
\hline
\end{tabular}




\subsection{Practice}

$58.4 \%$ of parents did not give the offered iron for their infants or stopped it within the first week of use. Of the remaining $41.6 \%$ who used iron for their infants, $59.4 \%$ of parents forgot to give it from time to time while $60.0 \%$ stopped using it for reasons other than forgetting.

Child refusal was considered by $55 \%$ of parents to be a legitimate reason to stop giving iron syrup and $63.0 \%$ of parents said that they would discontinue iron syrup if the infant developed bothersome side effects (Table 3).

$43.6 \%$ of parents brushed their infant's teeth after the use of iron syrup. Furthermore, only $23.0 \%$ of parents mixed iron syrup with juice and $69.1 \%$ did not mix it with anything (Table 4).

Table 3. Frequency and percent distribution of parent's general knowledge and practice towards iron syrup $(\mathrm{n}=$ 397)

\begin{tabular}{|c|c|c|c|c|c|c|c|c|c|c|}
\hline \multirow[t]{2}{*}{ General knowledge and practice } & \multicolumn{2}{|c|}{ Strongly agree } & \multicolumn{2}{|c|}{ Agree } & \multicolumn{2}{|c|}{$\begin{array}{l}\text { Neither agree } \\
\text { nor disagree }\end{array}$} & \multicolumn{2}{|c|}{ Disagree } & \multicolumn{2}{|c|}{$\begin{array}{l}\text { Strongly } \\
\text { disagree }\end{array}$} \\
\hline & $\mathbf{n}$ & $\%$ & $\mathbf{n}$ & $\%$ & $\mathbf{n}$ & $\%$ & $\mathbf{n}$ & $\%$ & n & $\%$ \\
\hline $\begin{array}{l}\text { Iron syrup is necessary to treat iron } \\
\text { deficiency anemia }\end{array}$ & 94 & $23.7 \%$ & 199 & $50.1 \%$ & 47 & $11.8 \%$ & 51 & $12.8 \%$ & 6 & $1.5 \%$ \\
\hline $\begin{array}{l}\text { The side effects of iron syrup are annoying } \\
\text { enough to be discontinued }\end{array}$ & 93 & $23.4 \%$ & 163 & $41.1 \%$ & 41 & $10.3 \%$ & 93 & $23.4 \%$ & 7 & $1.8 \%$ \\
\hline $\begin{array}{l}\text { Child refusal of iron syrup is annoying } \\
\text { enough to stop it }\end{array}$ & 72 & $18.1 \%$ & 149 & $37.5 \%$ & 41 & $10.3 \%$ & 122 & $30.7 \%$ & 13 & $3.3 \%$ \\
\hline $\begin{array}{l}\text { It is possible to get rid of the side effects of } \\
\text { iron if it is used in a proper way }\end{array}$ & 35 & $8.8 \%$ & 243 & $61.2 \%$ & 62 & $15.6 \%$ & 49 & $12.3 \%$ & 8 & $2.0 \%$ \\
\hline $\begin{array}{l}\text { Diet rich in iron is enough to replace the iron } \\
\text { therapy }\end{array}$ & 176 & $44.3 \%$ & 165 & $41.6 \%$ & 15 & $3.8 \%$ & 37 & $9.3 \%$ & 4 & $1.0 \%$ \\
\hline
\end{tabular}

Table 4. Frequency and percent distribution of parent's practice towards iron syrup $(n=165)$

\begin{tabular}{llll}
\hline & & $\mathbf{n}$ & \multicolumn{1}{c}{$\mathbf{0}$} \\
\hline Most of the time, do you brush your child teeth after using iron supplement? & Yes & 72 & $43.6 \%$ \\
& No & 45 & $27.3 \%$ \\
& No teeth & 48 & $29.1 \%$ \\
\hline If you give your child iron supplement, you mix it most of the time with: & Nothing & 114 & $69.1 \%$ \\
& Water & 8 & $4.8 \%$ \\
& Juice & 38 & $23.0 \%$ \\
& Milk & 4 & $2.4 \%$ \\
& Food & 1 & $0.6 \%$ \\
\hline
\end{tabular}

\section{Discussion}

Our study showed that the prevalence of anemia in infants aged 9 months who took part in this research was $45 \%$, which is in concordance with previous statistics and local studies(Ahmed G, September 2006; Al-Alawi F, March 2015; Al-Alawi M, 2014; Ministry of Health; WHO, 2015). This calls for attention to a treatable and preventable public health problem.

Overall, majority of parents correctly answered the knowledge questions regarding iron syrup side effects, whereas, only a small percentage of parents correctly answered the questions regarding IDA complications. This difference may be due to their past experience with iron syrup use, or the information acquired by parents regarding the side effects was more than the information acquired for complications. Moreover, the time needed for the side effects to be apparent is much shorter than that for IDA complications. These all may affect parental decisions in starting iron syrup for their infants. 
The comparison with other published literature was limited owing to different methodology, measurement tools and sampling techniques used.

Due to a difference in defining the acceptable treatment duration, a variation in results of the comparable studies was found. For instance, in our study more than half of parents (58.0\%) did not give the offered iron for their infants or stopped it within the first week of use. This is in accordance with a study conducted in Israel which reported as much as $60 \%$ of parents did not give the iron syrup to their infants on daily basis for a period of three months (Bilenko et al., 2007). On a national level, a study was conducted in one of the Health Centers in Bahrain which revealed that $92.4 \%$ of mothers either did not give or stopped the iron syrup within one month (Al-Alawi F, March 2015).

In our study, most parents would discontinue the iron syrup if the infant developed bothersome side effects. Besides that, it was evident that teeth-staining is a well-known side effect by most parents. Moreover, iron syrup is mistakenly thought by most parents to cause teeth decay. Both findings are in agreement with a regional study done in Iran (Talebi et al., 2012). This could explain the high percentage of teeth brushing reported in our study following the administration of iron treatment. Additionally, our research revealed that the majority of parents thought that an iron-rich diet can substitute iron therapy. This could explain parents' refusal to start iron therapy or their non-compliance with the treatment.

The large percentage of parents who are unaware of IDA complications especially heart problems (81.9\%), psychological and behavioral problem (82.6\%), and learning difficulty $(69.0 \%)$ might lead to underestimation of the seriousness of the disease and thus to non-compliance.

A significant proportion of parents thought that it is possible to overcome the iron syrup side effects. However, compared to a regional study conducted in Iran which showed $87.0 \%$ of parents mixing iron syrup with juice or water(Talebi et al., 2012), our study yielded a much lesser percentage (27.8\%). This could be due to a lack of proper parental education and guidance and/or the difference in research settings as the comparative study was conducted by dentists who may have given more attention to teeth-related problems, while this study was conducted by primary healthcare providers.

One of the strengths in this study is the random sampling technique employed, the trained interviewers, and it being the first study locally to explore parental knowledge and practice towards iron syrup, which may help in forming the basis for future research.

On the other hand, there are a few limitations as well. First of all, only public primary healthcare centers were included, whereas the private sector was not included, which might affect the external validity of our results. Secondly, the inaccurate and incomplete medical records were one of the main challenges faced. Thirdly, data collection took place during the holy month of Ramadan and the summer time when a lot of people were away. So, this could have led to a high number of unreachable parents and thus to selection bias. Recall and social desirability biases could also not be eliminated. Lastly, due to unavailability of a well-validated questionnaire and total scoring, individual questionnaire items were compared instead of the overall results.

The guidelines of IDA treatment among infants should be enforced through Continuous Medical Education or workshops for primary care physicians. Regular clinical audits would ensure complete and thorough medical records. Increasing the awareness of the public regarding IDA, its treatment and its complications should be emphasized through educational programs. Interventional research is needed to test the effectiveness of such educational intervention.

\section{Conclusion}

In conclusion, anemia is prevalent among 9-month-old infants with considerable gaps in knowledge and practices of parents. This study highlights some of the possible factors that may contribute to such gaps which could inform healthcare policy-makers to set better strategies for prevention and successful treatment.

\section{Funding}

This research did not receive any specific grant from funding agencies in the public, commercial, or not-for-profit sectors.

\section{Competing Interests Statement}

The authors declare that they have no competing or potential conflicts of interest.

\section{References}

Abdinia, B. (April 2014). Maternal knowledge and Performance about Use of Iron and Multivitamin Supplements 
in Children in Northwest, Tabriz-Iran. International Journal of Pediatrics, 2(2.2), 119-123.

Ahmed, G., Hashim, K., Al-Ekri, E., Al-Arrayed, O., \& Mandeel, M. (September 2006). Treatment of Anemia in Infants. Bahrain Medical Bulletin, 28(3).

Al-Alawi, F., Hashim, W., Abdulla, G., \& Al-Sayyad, A. (March 2015). The effect of nutritional educational intervention to mothers on improving the hemoglobin level of their nine month old children attending Ahmed Ali Kanoo Health Center in the Kingdom of Bahrain. World Family Medicine Journal, 13(2).

Al-Alawi, M., \& Sarhan, N. (2014). Prevalence of anemia among nine-month-old infants attending primary care in Bahrain. J Bahrain Med Soc, 25(1).

Bilenko, N., Yehiel, M., Inbar, Y., \& Gazala, E. (2007). The association between anemia in infants, and maternal knowledge and adherence to iron supplementation in southern Israel. Isr Med Assoc J, 9(7), 521-524.

Carter, R. C., Jacobson, J. L., Burden, M. J., Armony-Sivan, R., Dodge, N. C., Angelilli, M. L., ... Jacobson, S. W. (2010). Iron deficiency anemia and cognitive function in infancy. Pediatrics, 126(2), e427-434. doi:10.1542/peds.2009-2097. https://doi.org/10.1542/peds.2009-2097

Coutinho, G. G., Goloni-Bertollo, E. M., \& Pavarino-Bertelli, E. C. (2008). Effectiveness of two programs of intermittent ferrous supplementation for treating iron-deficiency anemia in infants: randomized clinical trial. Sao Paulo Med J, 126(6), 314-318. https://doi.org/10.1590/S1516-31802008000600004

Hassan, A. E., Kamal, M. M., Fetohy, E. M., \& Turky, G. M. (2005). Health education program for mothers of children suffering from iron deficiency anemia in United Arab Emirates. J Egypt Public Health Assoc, $80(5-6), 525-545$.

Lozoff, B., Clark, K. M., Jing, Y., Armony-Sivan, R., Angelilli, M. L., \& Jacobson, S. W. (2008). Dose-response relationships between iron deficiency with or without anemia and infant social-emotional behavior. $J$ Pediatr, 152(5), 696-702, 702 631-693. https://doi.org/10.1016/j.jpeds.2007.09.048

Ministry of Health, Kingdom Of Bahrain. Health Statistics 2013, Primary Health Care - Government Sector (pp. 18).

Sherriff. A., Emond, A., Bell, J. C., et al. (2001). Should infants be screened for anemia? A prospective study investigating the relation between hemoglobin at 8, 12, and 18 months and development at 18 months. Arch Dis Child(84:480). https://doi.org/10.1136/adc.84.6.480

Salehi, F., Abdollahi, Z., Ahadi, Z., Qorbani, M., Shafiee, G., Khosravi, S., ... Heshmat, R. (2015). The association between socioeconomic status with knowledge, attitude and practice toward use of iron and vitamin AD supplements among infants and pregnants: the NUTRI-KAP survey. Iranian Journal of Public Health, 44(1), $1-8$.

Talebi, M., Parisay, I., \& Mokhtari, N. (2012). The parents' knowledge and behavior towards the effects of using iron supplements on tooth staining and dental caries in Mashhad, Iran. Dent Res J (Isfahan), 9(6), 715-718.

UNICEF/UNU/WHO. (2001). Iron deficiency anemia: assessment, prevention, and control. A guide for programme managers. Geneva.

WHO. (2015). The global prevalence of anemia in 2011. Geneva: World Health Organization.

Williams, J., Wolff, A., Daly, A., MacDonald, A., Aukett, A., \& Booth, I. W. (1999). Iron supplemented formula milk related to reduction in psychomotor decline in infants from inner city areas: randomised study. $B M J$, 318(7185), 693-697. https://doi.org/10.1136/bmj.318.7185.693

\section{Copyrights}

Copyright for this article is retained by the author(s), with first publication rights granted to the journal.

This is an open-access article distributed under the terms and conditions of the Creative Commons Attribution license (http://creativecommons.org/licenses/by/4.0/). 